\title{
Multivariable relationship with the change of sulfate concentration and dynamic model for the water demand of the Huambi parish, Ecuador, South America
}

\author{
Edgar Guerrón Varela ${ }^{1,2}$, David Vinicio Carrera-Villacrés ${ }^{1,2}$, Christian Torres $^{2}$, Mario Barba ${ }^{2}$, Alexandra Monge $^{2}$ y \\ Jenny Hernández². \\ ${ }^{1}$ Universidad de las Fuerzas Armadas ESPE. Departamento de Ciencias de la Tierra y la Construcción y Departamento \\ de Ciencias Exactas. Av. General Rumiñahui s/n, Sangolquí, Ecuador, dvcarrera@espe.edu.ec \\ ${ }^{2}$ Universidad Central del Ecuador. Facultad de Ingeniería en Geología Minas Petróleos y Ambiental FIGEMPA. Carrera \\ de Ambiental. Av. Universitaria, Quito, Ecuador, dvcarrera@uce.edu.ec \\ Grupo de Investigación en Contaminación Ambiental (GICA)
}

\begin{abstract}
The growing population has generated the need for a greater amount of agrarian, water and land resources, this work present dynamic modeling that allows knowing the supply and demand of water for agricultural use in function to the population growth of the parish Huambi in Ecuador, South America. To the year 2039, there will be a water demand of $225,000,000 \mathrm{m3}$ year-1 which should satisfy an approximate population of 6,000 farmers.

However, by 2021, the offer will not cover the existing demand. In addition, correlation studies of the $R A S$ vs Mg+Bicarbonate, $R A S$ vs $C a+$ Bicarbonate, $R A S$ vs ISP, RAS vs EC+STD, of which the highest correlation was that of $R A S$ vs EC+STD with a ratio of 0.8596 , also determinate that the river has a stronger correlationship between $R A S$ and Ca+Bicarbonate than the one with magnesium. The potential salinity index (SP) shows values of 3.0 indicating that the water is optimal for irrigation. The low values in the sodium absorption ratio indicate a low probability of sodium exchange. Using the procedure of the US Department of Agriculture where the EC relates to the sodium adsorption ratio allow the results to be located in the C1S1 field, which corresponds to the water bodies of very good quality for irrigation. In conclusion, it was determinated that the water of the Arapicos River is suitable for irrigation, highlighting in all indicators used, despite its high quality this river is close to an excess of demand or shortage.
\end{abstract}

Keywords-Correlation, adsorption of Sodium, river Arapicos, Index of potential salinity.

Digital Object Identifier (DOI):

http://dx.doi.org/10.18687/LACCEI2020.1.1.328

ISBN: 978-958-52071-4-1 ISSN: 2414-6390

$18^{\text {th }}$ LACCEI International Multi-Conference for Engineering, Education, and Technology: "Engineering, Integration, and Alliances for a Sustainable Development" "Hemispheric Cooperation for Competitiveness and Prosperity on a Knowledge-Based Economy", July 27-31, 2020, Virtual Edition. 


\title{
Relación multivariable con el cambio de concentración de sulfatos y modelo dinámico para la demanda hídrica de la parroquia Huambi, Ecuador, Sur América
}

\author{
Edgar Guerrón Varela ${ }^{1,2}$, David Vinicio Carrera-Villacrés ${ }^{1,2}$, Christian Torres ${ }^{2}$, Mario Barba $^{2}$, Alexandra Monge ${ }^{2}$ y \\ Jenny Hernández 2 . \\ ${ }^{1}$ Universidad de las Fuerzas Armadas ESPE. Departamento de Ciencias de la Tierra y la Construcción y Departamento de \\ Ciencias Exactas. Av. General Rumiñahui s/n, Sangolquí, Ecuador, dvcarrera@espe.edu.ec \\ ${ }^{2}$ Universidad Central del Ecuador. Facultad de Ingeniería en Geología Minas Petróleos y Ambiental FIGEMPA. Carrera de \\ Ambiental. Av. Universitaria, Quito, Ecuador, dvcarrera@uce.edu.ec \\ Grupo de Investigación en Contaminación Ambiental (GICA)
}

\begin{abstract}
The growing population has generated the need for a greater amount of agrarian, water and land resources, this work present dynamic modeling that allows knowing the supply and demand of water for agricultural use in function to the population growth of the parish Huambi in Ecuador, South America. To the year 2039, there will be a water demand of 225,000,000 $\mathrm{m}^{3}$ year-1 which should satisfy an approximate population of 6,000 farmers.

However, by 2021, the offer will not cover the existing demand. In addition, correlation studies of the $R A S$ vs Mg+Bicarbonate, $R A S$ vs Ca+Bicarbonate, $R A S$ vs ISP, RAS vs EC+STD, of which the highest correlation was that of $R A S$ vs $E C+S T D$ with a ratio of 0.8596, also determinate that the river has a stronger correlationship between $R A S$ and Ca+Bicarbonate than the one with magnesium. The potential salinity index (SP) shows values of 3.0 indicating that the water is optimal for irrigation. The low values in the sodium absorption ratio indicate a low probability of sodium exchange. Using the procedure of the US Department of Agriculture where the EC relates to the sodium adsorption ratio allow the results to be located in the C1S1 field, which corresponds to the water bodies of very good quality for irrigation. In conclusion, it was determinated that the water of the Arapicos River is suitable for irrigation, highlighting in all indicators used, despite its high quality this river is close to an excess of demand or shortage.
\end{abstract}

Keywords-Correlation, adsorption of Sodium, river Arapicos, Index of potential salinity.

\section{INTRODUCCIÓN}

El incremento de las alteraciones de los cursos de agua y la sensibilidad a este problema por parte de los organismos competentes ha hecho que en todos los países desarrollados se realicen programas de control y vigilancia de la calidad de las aguas [1]. Gran parte de los parámetros utilizados para la evaluación de la calidad del recurso hídrico son de factores físico-químicos, y de manera especial basados en composición química [2.] Entre los compuestos más comunes que se pueden encontrar en aguas dulces se encuentran con un mayor porcentaje los carbonatos, bicarbonatos, sulfatos, cloruros entre otros [3]. Existen diferentes parámetros físico-químicos para determinar el estado de las aguas en cuestión agrícola y de consumo entre ellos se encuentran: $\mathrm{pH}, \mathrm{CE}$, sólidos totales, bicarbonatos, calcio, cloruros, magnesio, sulfatos, etc. La temperatura posee una gran importancia en el desarrollo de los diversos procesos que se efectúan en el agua, el $\mathrm{pH}$ influye en la acidez, basicidad o neutralidad, en las medidas de $\mathrm{pH}$ hay que tener presente que estas sufren variaciones con la temperatura. La conductividad eléctrica (CE) en el agua se comporta como aislante eléctrico siendo las sustancias en ella disueltas las que proporcionan al agua la capacidad de conducir la corriente eléctrica [4]. La dureza del agua está relacionada con la cantidad de cationes metálicos, excepto metales alcalinos, que están presentes en ella y que pueden existir como carbonatos o bicarbonatos. Entre estos metales se encuentran el calcio, magnesio, hierro, bario, estroncio [5]. La dinámica fluvial es un conjunto de procesos complejos activos $\mathrm{y}$ metamorfosis de los sistemas fluviales tanto en su componente espacial como en su evolución temporal. Los ríos son entidades dinámicas que evolucionan por sí mismos (factores hidrológicos, meteorológicos y geomorfológicos); además sus cambios se ven afectados por la intervención humana (factores antrópicos). La interrupción y la contaminación de los ríos pueden modificar y alterar el flujo normal del ciclo hidrológico, provocando serios trastornos en el clima de la tierra, en los procesos isostáticos y en la preservación de diversos ecosistemas [6]. Para lograr una comprensión del comportamiento de los ríos, es necesario realizar una definición y un estudio hidrológico [7].

Los ecosistemas de agua dulce están sometidos a varios factores naturales que inciden en el aumento de sus niveles de salinidad, como son la geología de los cauces sobre los que se asientan o la climatología [8].

Los volúmenes disponibles de agua dulce para uso agrícola y urbano a nivel mundial han disminuido notablemente, esto es consecuencia del uso excesivo de aguas superficiales y subterráneas destinadas al riego agrícola para la producción de alimentos de una población en constante crecimiento [9]. En la actualidad las fuentes disponibles de agua no pueden satisfacer la creciente demanda [10]. Las necesidades de agua para riego dependen de las exigencias hídricas de los cultivos y del agua que está disponible para los mismos de forma natural [11]. La clasificación de agua para

\footnotetext{
Digital Object Identifier (DOI):

http://dx.doi.org/10.18687/LACCEI2020.1.1.328

ISBN: 978-958-52071-4-1 ISSN: 2414-6390
}

$\mathbf{1 8}^{\text {th }}$ LACCEI International Multi-Conference for Engineering, Education, and Technology: "Engineering, Integration, and Alliances for a Sustainable Development" "Hemispheric Cooperation for Competitiveness and Prosperity on a Knowledge-Based Economy", July 27-31, 2020, Virtual Edition. 
riego de cultivos toma en cuenta la peligrosidad sódica y salina a partir del valor de CE expresada en $\mu \mathrm{S} / \mathrm{cm}$ y del índice RAS expresado en miliequivalentes [12].

Con el análisis de (pH, CE, sólidos totales, bicarbonatos, calcio, cloruros, magnesio, sulfatos), se logra determinar la calidad del agua en la parroquia Huambi del cantón Sucúa, siendo esta la segunda más habitada con 2891 habitantes con un pasado ancestral Shuar, la parroquia se abastece de 3 ríos, Tutanangoza, Upano y Arapicos, el dinamismo productivo de la parroquia Huambi está orientado principalmente a actividades primarias desarrolladas en el sector agropecuario [13], [14],[15].

Huambi parroquia del cantón Sucúa, posee problemáticas de aprovechamiento del agua, y desconocimiento de la calidad y cantidad de esta para el uso en sus actividades primarias como es la agricultura. En este trabajo se tiene como objetivo determinar la correlación de distintos parámetros(temperatura, $\mathrm{pH}, \mathrm{CE}$, calcio, magnesio, sodio, cloruros, sulfatos) mismos que se obtuvieron mediante análisis físico-químicos realizados en las muestras de agua del río Arapicos, así como la creación de un modelo dinámico utilizando el software Vensim para la gestión hídrica por medio de datos de evapotranspiración, mediciones de caudales y tasa de natalidad y mortalidad, determinando la cantidad de agua aprovechable para los cultivos y el beneficio de la población. La calidad del recurso hídrico se logró determinar por medio de la relación de adsorción de sodio (RAS) y salinidad (SP)con información de Riverside mediante el software R se da la correlación lineal de Pearson existente entre los factores (RAS y CE).

\section{MATERIALES Y MÉTODOS}

\section{A. Determinación de la zona de estudio}

La parroquia rural Huambi perteneciente al cantón Sucúa de la provincia de Morona Santiago (Fig. 1), actualmente consta de una población de 2891 personas según el último censo en el año 2010. La parroquia rural Huambi tiene una superficie de $214.82 \mathrm{~km}^{2}$ [16] es importante mencionar que Huambi se encuentra próxima a los ríos Arapicos, y Tutanangoza.

TABLA I

UBICACIÓN DE LOS PUNTOS DE MUESTREO Y NUMERO DE MUESTRAS TOMADAS POR PUNTO

\begin{tabular}{ccc|cc}
\hline \multirow{2}{*}{$\begin{array}{c}\text { Número } \\
\text { de } \\
\text { muestras }\end{array}$} & $\begin{array}{c}\text { Puntos de } \\
\text { Referencia }\end{array}$ & \multicolumn{2}{c}{$\begin{array}{c}\text { Cbicación en } \\
\text { UTM WGS84 }\end{array}$} & $\begin{array}{c}\text { Altura } \\
(\mathrm{msnm})\end{array}$ \\
\hline M1 & Río & 813047 & 9718742 & 764 \\
M2 & Fuente Subterránea & 814214 & 9717718 & 707 \\
M3 & Fuente Subterránea & 814288 & 9717856 & 697 \\
M4 & Cocha 1 & 815026 & 9727545 & 715 \\
M5 & Cocha 2 & 815082 & 9727600 & 710 \\
\hline
\end{tabular}

Fuente: Los autores
Los puntos de muestreo fueron dos cochas de aglomeración de aguas lluvia cercanas al río Arapicos, un punto aguas arriba, y dos puntos de fuentes de agua subterránea cercanas a

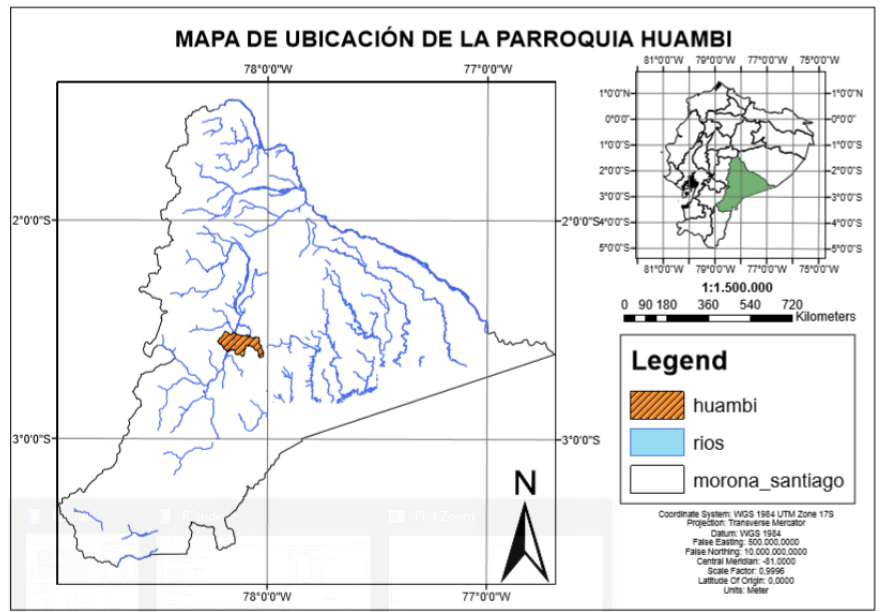

Fig. 1 Ubicación de la parroquia Huambi (Fuente: Los autores)

la unión entre el río Arapicos y el río Tutanangoza (Tabla I). Se analizaron parámetros fisicoquímicos de acuerdo con los estándares expuestos en la normativa nacional (NTE-INEN0973; EPA 1983, 1701; NOMAA-93-1984; Standard Methods, HACH 2000) en los laboratorios de ingeniería ambiental de la Facultad de Ingeniería en Geología, Minas, Petróleos y Ambiente.

TABLA II

DETERMINACIONES FISICOQUIMICAS DE LAS MUESTRAS DE AGUA (FUENTE: LOS AUTORES)

\begin{tabular}{|c|c|c|c|}
\hline Parámetro & Unidad & Equipo & Referencia \\
\hline $\mathrm{pH}$ & & $\begin{array}{l}\text { pH-metro marca } \\
\text { HANNA modelo } \\
\text { HI2210-01 }\end{array}$ & $\begin{array}{l}\text { Standard Methods } \\
4500-H^{\oplus}[19]\end{array}$ \\
\hline
\end{tabular}

\begin{tabular}{|c|c|c|c|}
\hline Temperatura & ${ }^{\circ} \mathrm{C}$ & & $\begin{array}{l}\text { Standard Methods } \\
2550 \mathrm{~B}[20]\end{array}$ \\
\hline $\begin{array}{l}\text { Sólidos Totales } \\
\text { Disueltos }\end{array}$ & Ppm & $\begin{array}{l}\text { Estufa SLN } 115 \\
\text { marca POL-EKD } \\
\text { APARATURA }\end{array}$ & $\begin{array}{l}\text { NOM-AA-341981 } \\
{[21]}\end{array}$ \\
\hline $\begin{array}{l}\text { Conductividad } \\
\text { Eléctrica }\end{array}$ & $\mathrm{uS} / \mathrm{cm}$ & $\begin{array}{l}\text { Conductímetro de } \\
\text { mesa marca } \\
\text { Thermo Scientific } \\
\text { Orion Star A212 }\end{array}$ & $\begin{array}{l}\text { Standard Methods } \\
2510 \text { B [22] }\end{array}$ \\
\hline $\begin{array}{l}\text { Cationes } \\
\left(\mathrm{Ca}^{+2}\right)\end{array}$ & $\mathrm{mg} / \mathrm{L}$ & & $\begin{array}{l}\text { Standard Methods } \\
3500-\mathrm{Ca}^{+2}-\mathrm{B} \text { [23] }\end{array}$ \\
\hline $\begin{array}{l}\text { Cationes } \\
\left(\mathrm{Mg}^{+2}\right)\end{array}$ & $\mathrm{mg} / \mathrm{L}$ & & $\begin{array}{l}\text { Standard Methods } \\
3500-\mathrm{Mg}^{+2}-\mathrm{B}[24]\end{array}$ \\
\hline $\begin{array}{l}\text { Aniones } \\
\left(\mathrm{SO}_{4}^{-2}\right)\end{array}$ & $\mathrm{mg} / \mathrm{L}$ & Titulación & $\begin{array}{l}\text { Standard Methods } \\
4500-\mathrm{SO}_{4}{ }^{-2}-\mathrm{E}[25]\end{array}$ \\
\hline Aniones $\left(\mathrm{Cl}^{-}\right)$ & $\mathrm{meq} / \mathrm{L}$ & Titulación & $\begin{array}{l}\text { Standard Methods } \\
250-\mathrm{Cl}^{-} \text {- B [26] }\end{array}$ \\
\hline
\end{tabular}




\begin{tabular}{lccc}
$\begin{array}{l}\text { Aniones } \\
\left(\mathrm{HCO}_{2}{ }^{-2}\right)\end{array}$ & $\mathrm{meq} / \mathrm{L}$ & Titulación & $\begin{array}{l}\text { APHA método } \\
2330-\mathrm{B}, \mathrm{D}[27]\end{array}$ \\
$\begin{array}{l}\text { Aniones } \\
\left(\mathrm{CO}_{2}{ }^{-2}\right)\end{array}$ & $\mathrm{meq} / \mathrm{L}$ & Titulación & \\
\hline
\end{tabular}

Los resultados de los análisis de laboratorio $(\mathrm{pH}$, Conductividad, Calcio, Magnesio, Carbonatos, Bicarbonatos, Cloruros y Sulfatos) se los tabularon (Tabla II) y mediante el software R usando la librería "Performance Analytics" de donde se obtiene una colección de funciones para análisis de desempeño, riesgo, correlaciones y regresiones [17]. Con esta información se procedió a realizar el análisis de las regresiones lineal del comportamiento de las variables analizadas en laboratorio, por medio del coeficiente de correlación de Pearson, coeficiente de determinación $\mathrm{R}^{2}$ y el error estándar [18].

\section{B. Relación de adsorción de sodio (RAS)}

Para la determinación de la relación de adsorción de sodio se lo hizo mediante la ecuación (1), con los datos de las concentraciones de catiónicas de $\mathrm{Ca}^{2+}, \mathrm{Mg}^{2+}$ y Na ${ }^{+}$en meq/L, ya que el RAS en sus diferentes conceptualizaciones ha sido ampliamente satisfactorio para el manejo de suelos y aguas salinas [27].

$$
R A S=\frac{N a^{+}}{\sqrt{\left(C a^{2+}+M g^{2+}\right) / 2}}
$$

Para el estudio de la correlación entre RAS y sulfatos presentes en las aguas muestreadas se procedió a utilizar el software estadístico "R Studio" [29].

\section{Población de la parroquia Huambi}

Para un análisis realista del crecimiento poblacional se consideró el Plan de Desarrollo y Ordenamiento Territorial de la Parroquia Huambi, (2015) menciona que la tasa de natalidad en la parroquia considerada es de $10,50 \%$, dicho crecimiento se ve afectado por la tasa de mortalidad $3,61 \%$ [13].

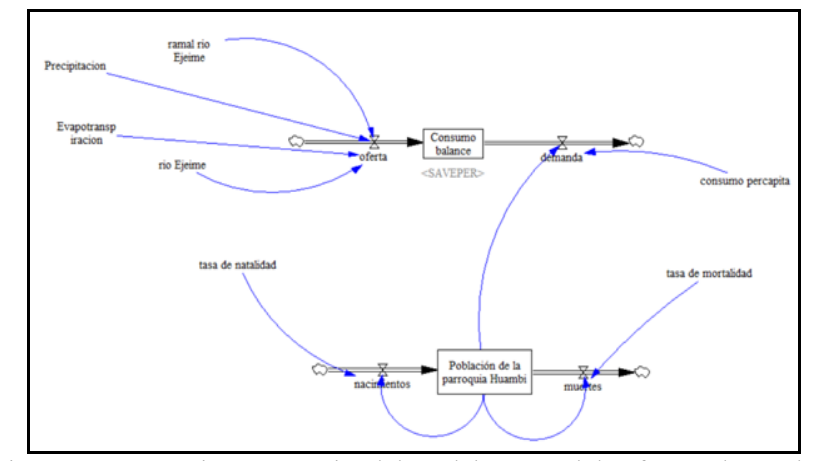

Fig. 2 Representación en Vensim del modelo general de oferta y demanda para uso agrícola (Fuente: Los autores)
En el área de intervención se analizó la demanda del recurso hídrico que los agrícolas de la parroquia Huambi requieren para su abastecimiento y mantener sus cultivos en un periodo de 20 años; se utilizó el software libre Vensim 7.3.4 para el desarrollo del modelamiento dinámico de crecimiento poblacional y el balance de consumo. Las variables utilizadas se interrelacionan llevando a cabo un análisis complejo de datos, esto a su vez genera un balance entre la disponibilidad de agua en el Río Arapicos y la demanda de agua para satisfacer el consumo de las actividades agrícolas de esta población con una proyección estimada de 20 años. Es importante mencionar que el modelo se ajustó a la realidad de la vertiente que integra el Río Arapicos, se tomaron datos proporcionados por el INAMHI de las estaciones meteorológicas M1040, M0497 Y M0501, entre ellas la precipitación promedio anual en de cada una, así también como la evapotranspiración que los anuarios meteorológicos nos proporcionaban, así como el aporte de uno de los ramales del Río Ejeime y el Río Ejeime como tal, para poder determinar la oferta de agua existente en el Río Arapicos dando un total de $7.0727 \times 10^{9}[28]$.

\section{E. Arquitectura del modelo para abastecimiento del poblado de Huambi}

Este modelo consideró la información consolidada de la oferta demanda de agua de cada una de las aportaciones al Río Arapicos; quebradas, precipitación, evapotranspiración, al igual que las captaciones de adicionales; el consumo percápita; la dinámica poblacional dada por los nacimientos y defunciones, en contraste con la tasa de mortalidad y la esperanza de vida (Fig. 2).

A continuación, se presenta una tabla con las variables que fueron consideradas en el modelo dinámico para abastecimiento de agua potable al poblado de Huambi.

\section{F. Balance de consumo}

El balance de consumo está constituido por la oferta y demanda del recurso hídrico. La demanda resulta de la relación entre la población de la parroquia y el consumo per cápita de acuerdo con el Ordenamiento Territorial y Plan de Desarrollo de Río Blanco [29].

La forma de expresión de este balance de masa fue dada por una ecuación diferencial, que describió la tasa de cambio de la variable caudal en un determinado tiempo (ecuación 2).

$$
\frac{d Q}{d t}=f(Q, t)
$$

Donde $Q$ significa el caudal en $1 \operatorname{seg}^{-1}$ y $f(Q, t)$ es la fórmula de la que depende el caudal en el tiempo y que en contexto fue entendida como:

TABLA III

VARIABLES TOMADAS EN CUENTA PARA EL MODELO RELACIONAL (FUENTE: LOS AUTORES)

\section{Modelo dinámico}




\begin{tabular}{|c|c|c|c|c|}
\hline Variable & Unidades & $\begin{array}{l}\text { Descripción y fuente } \\
\text { de información }\end{array}$ & $\begin{array}{l}\text { Valor en } \\
\text { el } \\
\text { modelo }\end{array}$ & $\begin{array}{l}\text { Tipo de } \\
\text { variable }\end{array}$ \\
\hline $\begin{array}{l}\text { Balance de } \\
\text { consumo }\end{array}$ & $\mathrm{m}^{3}$ & $\begin{array}{l}\text { Balance de Consumo que } \\
\text { resulta de la resta entre la } \\
\text { oferta y la demanda de } \\
\text { agua del Río Arapicos }\end{array}$ & & Variable \\
\hline $\begin{array}{l}\text { Consumo } \\
\text { Percápita } \\
\text { anual }\end{array}$ & $\mathrm{m}^{3} /$ persona & $\begin{array}{c}\text { La estimación del } \\
\text { consumo Per-capita de } \\
\text { agua se utilizó } \\
\text { Los datos otorgados por } \\
\text { INEN [28] }\end{array}$ & 0,18 & Constante \\
\hline $\begin{array}{l}\text { Precipitación } \\
\text { media } \\
\text { anual }\end{array}$ & $\mathrm{m}^{3} / \mathrm{m}^{2}$ & $\begin{array}{l}\text { La precipitación media } \\
\text { anual se obtuvo de } \\
\text { INAMHI [29] de una } 1 \\
\text { año en las estaciones } \\
\text { M1040, M0497, M0501 }\end{array}$ & 0,846 & Variable \\
\hline $\begin{array}{l}\text { Captación } \\
\text { Ramal Río } \\
\text { Ejeime }\end{array}$ & $\mathrm{m}^{3} /$ año & $\begin{array}{l}\text { La captación del Rio se } \\
\text { obtuvo de la secretaria } \\
\text { nacional de agua, } \\
\text { diagnóstico de } \\
\text { estadísticas. }\end{array}$ & $6,03^{\times} 10^{8}$ & Constante \\
\hline $\begin{array}{l}\text { Captación Río } \\
\text { Ejeime }\end{array}$ & $\mathrm{m}^{3}$ & $\begin{array}{l}\text { La captación del Rio se } \\
\text { obtuvo de la secretaria } \\
\text { nacional de agua, } \\
\text { diagnóstico de } \\
\text { estadísticas. }\end{array}$ & $6,40^{\times} 109$ & Constante \\
\hline $\begin{array}{l}\text { Oferta de agua } \\
\text { anual }\end{array}$ & $\mathrm{m}^{3} /$ persona & $\begin{array}{c}\text { La oferta de agua resulta } \\
\text { de la producción agrícola } \\
\text { per-cápita multiplicado } \\
\text { por la población agrícola } \\
\text { de Huambi }\end{array}$ & $6,59^{\times} 10^{7}$ & Constante \\
\hline $\begin{array}{l}\text { Tasa de } \\
\text { natalidad } \\
\text { anual }\end{array}$ & & Nacimientos al año & $2,50 \%$ & Variable \\
\hline $\begin{array}{l}\text { Tasa de } \\
\text { mortalidad } \\
\text { anual }\end{array}$ & & $\begin{array}{c}\text { Cuantos años en promedio } \\
\text { vive la gente }\end{array}$ & $0,103 \%$ & Variable \\
\hline $\begin{array}{c}\text { Población } \\
\text { Parroquia } \\
\text { Huambi }\end{array}$ & Personas & $\begin{array}{l}\text { Valor inicial de la } \\
\text { población } \\
\text { económicamente activa de } \\
\text { la parroquia Huambi }\end{array}$ & 1569 & Constante \\
\hline
\end{tabular}

Fuente: Los autores

$$
\frac{d Q}{d t}=Q_{\text {afluentes }}-Q_{\text {demanda }}=0
$$

Cabe destacar que este balance se igualó a cero, debido que el sistema presenta un equilibrio entre las entradas de agua asignado a la demanda y la oferta. Lo anterior se interpreta como que la tasa de cambio de la variable caudal es cero. Así, desde las consideraciones anteriores, fue posible establecer las relaciones para las respectivas aportaciones a la red hídrica que conforma el poblado de Huambi

\section{RESULTADOS Y DISCUSIÓN}

\section{A. Aptitud de agua para riego}

Los resultados obtenidos de los análisis de laboratorio se los expone a través de la Tabla 4. En dicha tabla podemos observar que el $\mathrm{pH}$ de las muestras se mantiene variando ligeramente la neutralidad, así mismo podemos observar a simple vista que son aguas con baja conductividad y concentración de sales y sales disueltas totales que no llegan a superar la unidad. A pesar de no encontrar valores que despunten dentro de la tabla es importante el analizar los valores de $\mathrm{pH}$ ya que de esto dependerá de las concentraciones relativas de especies disueltas de carbonato [30] lo cual se lo valida al no existir presencia de carbonatos debido a que no hay $\mathrm{pH}$ 's superiores a 8,5 .

\begin{tabular}{|c|c|c|c|c|c|c|c|c|c|}
\hline \multirow{3}{*}{$\begin{array}{l}\text { Parámet/ } \\
\text { Expresa }\end{array}$} & \multirow{3}{*}{ Uni. } & \multicolumn{5}{|c|}{$\begin{array}{c}\text { TABLA IV } \\
\text { ANÁLISIS FISICOQUÍMICO }\end{array}$} & & & \\
\hline & & \multicolumn{8}{|c|}{$\begin{array}{c}\text { Número de muestra de aguas analizadas, de los parámetros } \\
\text { fisicoquímico }\end{array}$} \\
\hline & & $\begin{array}{c}\text { M1- } \\
\text { A }\end{array}$ & $\begin{array}{c}\text { M1- } \\
\text { B }\end{array}$ & M2- & $\begin{array}{l}\text { M2- } \\
\text { B }\end{array}$ & M3- & $\begin{array}{c}\text { M3- } \\
\text { B }\end{array}$ & M4 & M5 \\
\hline $\mathrm{pH}$ & - & 7,44 & 7,30 & 6,35 & 6,16 & 6,22 & 6,35 & 7,32 & 6,83 \\
\hline $\begin{array}{l}\text { Conductividad } \\
\text { Eléctrica }\end{array}$ & $\mathrm{uS} / \mathrm{cm}$ & 69,6 & 74,1 & 91,70 & 91,01 & 74,5 & 72,4 & 81,8 & 19,7 \\
\hline STD & Ppm & 0,13 & 0,12 & 0,002 & 0,002 & 0,14 & 0,104 & 0,00 & 0,00 \\
\hline Temperatura & ${ }^{\circ} \mathrm{C}$ & 22,2 & 21,7 & 25,2 & 24,8 & 25,3 & 25,0 & 20,4 & 21,3 \\
\hline $\begin{array}{l}\text { Cationes } \\
\left(\mathrm{Ca}^{2+}\right)\end{array}$ & $\mathrm{meq} / \mathrm{L}$ & 23,3 & 26,7 & 22,0 & 22,3 & 21,3 & 18,0 & 60,0 & 4,80 \\
\hline $\begin{array}{c}\text { Cationes } \\
\left(\mathrm{Mg}^{2+}\right)\end{array}$ & $\mathrm{meq} / \mathrm{L}$ & 11,7 & 48,3 & 17 & 19,7 & 22 & 4 & 0 & 65,2 \\
\hline $\begin{array}{l}\text { Aniones } \\
\left(\mathrm{SO}_{4}^{2-}\right)\end{array}$ & $\mathrm{meq} / \mathrm{L}$ & 5,2 & 5,4 & 6,3 & 1,6 & 7,4 & 0,9 & 15,3 & 3,5 \\
\hline $\begin{array}{l}\text { Aniones } \\
\left(\mathrm{Cl}^{-}\right)\end{array}$ & $\mathrm{meq} / \mathrm{L}$ & 3,5 & 2 & 3,2 & 3 & 8 & 8 & 12 & 4 \\
\hline $\begin{array}{l}\text { Aniones } \\
\left(\mathrm{HCO}_{3}^{2-}\right)\end{array}$ & $\mathrm{meq} / \mathrm{L}$ & 2 & 1,67 & 1,2 & 1,35 & 0,4 & 0,4 & 16 & 12 \\
\hline $\begin{array}{l}\text { Aniones } \\
\left(\mathrm{CO}^{2-}{ }_{3}\right)\end{array}$ & $\mathrm{meq} / \mathrm{L}$ & 0,00 & 0,00 & 0,00 & 0,00 & 0,00 & 0,00 & 0,00 & 0,00 \\
\hline DQO & $\mathrm{meq} / \mathrm{L}$ & 8,30 & 8,30 & 7,80 & 7,80 & 7,40 & 7,40 & 0,00 & 0,00 \\
\hline
\end{tabular}

Fuente: Los autores

\section{B. Cálculo de la Relación de Adsorción de Sodio}

Para el cálculo de la relación de adsorción de sodio se procedió a pasar los resultados del laboratorio de $\mathrm{mg} \mathrm{l}^{-1}$ a meq $\mathrm{l}^{-1}$, obteniendo los resultados presentados en la Tabla V.

TABLA V

RELACIÓN DE ADSORCIÓN DE SODIO (FUENTE: LOS AUTORES)

\begin{tabular}{|c|c|c|c|c|c|c|c|c|c|}
\hline $\begin{array}{l}\text { Parámetro/ } \\
\text { Expresado }\end{array}$ & & \multicolumn{8}{|c|}{$\begin{array}{l}\text { Número de muestra de aguas para el cálculo de la } \\
\text { relación de adsorción de sodio. }\end{array}$} \\
\hline & & $\begin{array}{l}\text { M1 } \\
-\mathrm{A}\end{array}$ & $\begin{array}{l}\text { M1 } \\
\text {-B }\end{array}$ & $\begin{array}{l}\text { M2 } \\
-\mathrm{A}\end{array}$ & $\begin{array}{l}\text { M2 } \\
\text {-B }\end{array}$ & $\begin{array}{c}\text { M3 } \\
\text {-A }\end{array}$ & $\begin{array}{l}\text { M3 } \\
-B\end{array}$ & M4 & M5 \\
\hline RAS & $\begin{array}{c}\text { ueq/ } \\
\text { L }\end{array}$ & 70 & 44 & 65 & 62 & 60 & 92 & 00 & 43 \\
\hline
\end{tabular}

Fuente: Los autores

A partir de los datos calculados realizados podemos observar que en promedio las muestras de agua tomadas manejan un valor RAS de 0,055 , valor bastante bajo lo cual le produce una baja probabilidad de intercambio de sodio, lo que resulta beneficioso para su uso en el riego agrícola [30] esto se analizó al contrastar los resultados obtenidos del RAS con los datos de la Tabla VI. 
TABLA VI

CLASIFICACIÓN DE LAS AGUAS EN BASE AL R.A.S. [31]

\begin{tabular}{cccc} 
Clasificación & Tipo & R.A.S. & Observaciones \\
\hline S1 & Aguas bajas en Na & $<10$ & $\begin{array}{c}\text { Poca probabilidad de Psi } \\
\text { Presenta problemas si no } \\
\text { hay lavado }\end{array}$ \\
S2 & Aguas medias en Na & $10-18$ & $\begin{array}{c}\text { Necesita prácticas } \\
\text { especiales de manejo }\end{array}$ \\
S3 & Aguas altas en Na & $18-26$ & $\begin{array}{c}\text { Inadecuada excepto cuando } \\
\text { su salinidad es baja }\end{array}$ \\
S4 & $\begin{array}{c}\text { Aguas muy altas en } \\
\text { Na }\end{array}$ & $>26$ &
\end{tabular}

Fuente: Los autores

Por lo tanto, el agua del río Arapicos al ser utilizado para riego no representará problemas en los cultivos debido a su baja probabilidad de intercambio de sodio, que al presentar valores tan bajos se podría decir que simplemente no existirá intercambio de sodio, permitiendo así un crecimiento normal de los cultivos.

\section{Cálculo del Índice de salinidad potencial}

Para el cálculo del índice de salinidad potencial se utilizó los datos obtenidos en laboratorio transformado las unidades de g $^{1^{-1}}$ a meq $\mathrm{l}^{-1}$, se logró la Tabla VII.

TABLA VII

ÍNDICE DE SALINIDAD POTENCIAL

\begin{tabular}{|c|c|c|c|c|c|c|c|c|c|}
\hline $\begin{array}{l}\text { Parámetro/ } \\
\text { Expresado }\end{array}$ & & \multicolumn{8}{|c|}{$\begin{array}{l}\text { Número de muestra de aguas para el cálculo del índice de } \\
\text { salinidad potencial }\end{array}$} \\
\hline & & $\begin{array}{c}\text { M1- } \\
\text { A }\end{array}$ & $\begin{array}{c}\text { M1- } \\
\text { B }\end{array}$ & $\begin{array}{c}\text { M2- } \\
\text { A }\end{array}$ & $\begin{array}{c}\text { M2- } \\
\text { B }\end{array}$ & $\begin{array}{c}\text { M3- } \\
\text { A }\end{array}$ & $\begin{array}{c}\text { M3- } \\
\text { B }\end{array}$ & M4 & M5 \\
\hline SP & $\mathrm{meq} / \mathrm{l}$ & $\begin{array}{r}0,15 \\
4\end{array}$ & $\begin{array}{r}0,11 \\
7\end{array}$ & $\begin{array}{r}0,15 \\
7\end{array}$ & $\begin{array}{r}0,10 \\
3\end{array}$ & $\begin{array}{r}0,30 \\
5\end{array}$ & $\begin{array}{r}0,03 \\
2\end{array}$ & $\begin{array}{r}0,5 \\
03\end{array}$ & 0,150 \\
\hline
\end{tabular}

Fuente: Los autores

A partir de los datos calculados realizados podemos observar que en promedio las muestras de agua tomadas manejan un valor SP de 0,190 que es un valor bueno ya que esta agua no puede ser perjudicial a los cultivos debido al incremento de la presión osmótica del suelo [9] en referencia a la Tabla VIII.

TABLa VIII

SALINIDAD POTENCIAL [9]

\begin{tabular}{cc}
\hline Clase & Salinidad potencial en meq/l \\
\hline Buena & $<3.0$ \\
Condicionada & $3.0-15.0$ \\
No recomendable & $>15$ \\
\hline
\end{tabular}

Fuente: Los autores

\section{Cálculo de correlaciones.}

Para el cálculo de la correlación entre las variables se utilizó 4 indicadores estadísticos en donde cada uno nos indicó distintos comportamientos. En la Tabla IX podemos observar un coeficiente de determinación $\mathrm{R}^{2}$, este indicador de bondad de ajuste, nos indica que la proporción de la variación de la variable dependiente observada puedes ser explicada por el modelo de regresión simple.

\begin{tabular}{ccccc}
\multicolumn{6}{c}{ TABLA IX } \\
\hline RESULTADOS OBTENIDOS CON R ESTUDIO \\
\hline $\begin{array}{c}\text { RAS vs } \\
\text { estadísticos }\end{array}$ & $\begin{array}{c}\text { RAD + } \\
\text { C.E }\end{array}$ & $\begin{array}{c}\text { ISP } \\
\text { RAS vs } \\
\text { Ca+HCO }\end{array}{ }^{2}$ & $\begin{array}{c}\text { RAS vs } \\
\mathrm{MCO}_{3}{ }^{2}\end{array}$ \\
\hline $\begin{array}{c}\text { Residual } \\
\text { standard error }\end{array}$ & 0.01007 & 0.01681 & 0.01344 & 0.01344 \\
R squared & 0.8596 & 0.6649 & 0.7499 & 0.5857 \\
\hline
\end{tabular}

Fuente: Los autores

Para una mejor interpretación de los resultados obtenidos mediante el software Rstudio podemos observar la interpretación de los valores presentada en la Tabla X.

TABLA X

INTERPRETACIÓN DE LOS COEFICIENTES DE CORRELACIÓN [32]

\begin{tabular}{cc} 
Valor & Significados \\
\hline $\mathrm{R}=1$ & Correlación perfecta \\
$0.8<\mathrm{r}<1$ & Correlación muy alta \\
$0.6<\mathrm{r}<0.8$ & Correlación alta \\
$0.4<\mathrm{r}<0.6$ & Correlación moderada \\
\hline
\end{tabular}

\section{A. Interpretación de las gráficas}

Usando el software Rstudio se obtuvo mediante el paquete chart.correlation obteniendo la figura 3 , en la cual se puede observar los resultados gráficos de las correlaciones de las variables estudiadas
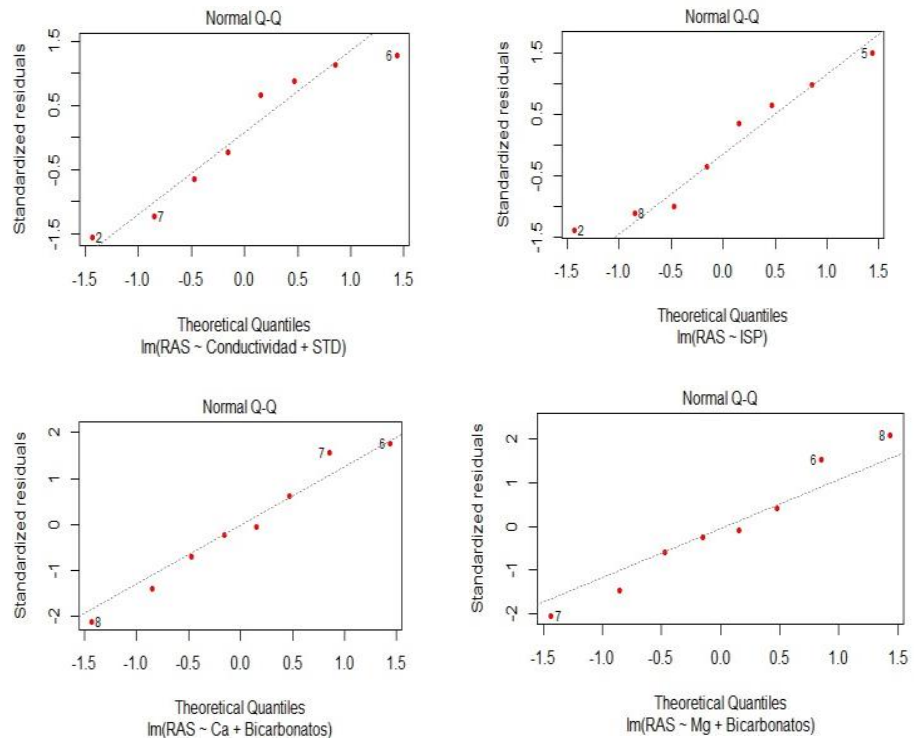

Fig. 3 Gráfico de la Distribución Normal Q-Q para las diferentes correlaciones realizadas

Al analizar los datos obtenidos de la tabla IX, se puede observar que existe una mayor correlación al analizar el RAS con los sólidos disueltos totales y la conductividad eléctrica que tiene una correlación de 0.8596 , lo que nos indica que la 
relación de adsorción de sodio puede afectar directamente conductividad eléctrica.

Al analizar la correlación de RAS vs ISP vemos que hay una alta correlación negativa.

Finalmente, se analizó la correlación entre RAS vs $\mathrm{Ca}+$ $\mathrm{HCO} 32$ y $\mathrm{Mg}+\mathrm{HCO} 32$ con lo que podría observarse que el RAS está mucho más afectado por la variación del bicarbonato de calcio que los del magnesio.

\section{B. Clasificación del agua de acuerdo con las normas \\ Riverside.}

Las normas Riverside, son aquellas con las que se maneja el departamento de agricultura de los Estados Unidos para determinar la calidad del agua en función de la relación de adsorción de sodio y la conductividad eléctrica. [26] Como podemos observar en la figura 4 donde los puntos se encuentran en el campo C1S1, cuya correspondencia nos indica que es agua de buena calidad para riego.

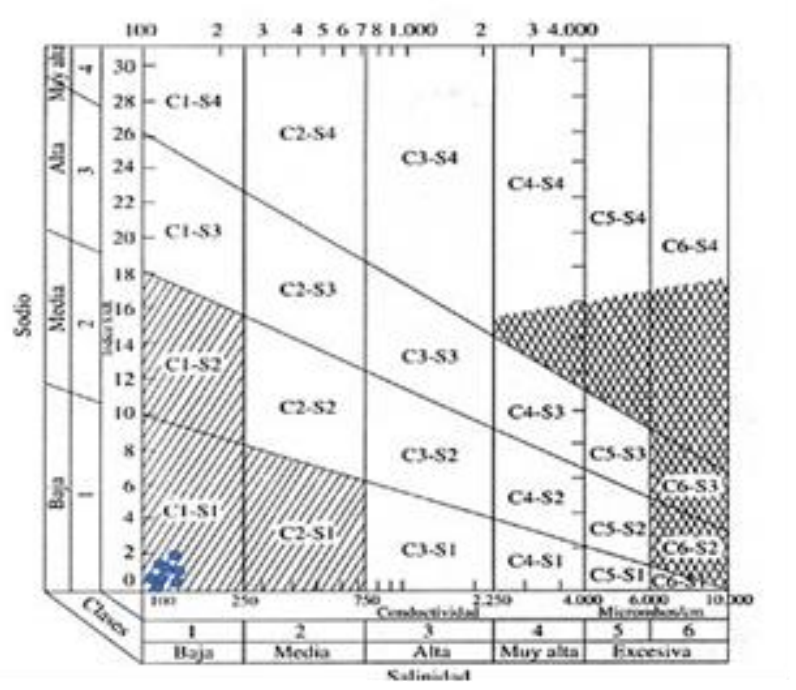

Fig. 4 Diagrama para la clasificación de la calidad del agua para riego de las normas Riverside

\section{Simulación del modelo para el año 2039}

De acuerdo al gráfico de la demanda vs la población muestra que el río Arapicos presenta una demanda creciente, en el 2039 se necesitará $225000 \frac{m^{3}}{\text { âी }}$. Lo mismo sucede para la población de agricultores en la parroquia Huambi crece de 1500 a 6000 agricultores/año, representado una alta demanda en un corto tiempo. Esto indica que los agricultores requerirán capacitaciones para el uso racional del agua, así como planes para la distribución equitativa del recurso hídrico.

En cuanto a lo observado en las gráficas de relación entre la oferta y la demanda la dinámica de abastecimiento de agua para la agricultura en la parroquia de Huambi podemos observar que la demanda se mantiene en el tiempo puesto que no se han considerado pérdidas tanto de, evaporación, escorrentía, teniendo una oferta aproximada de 6.5 Billones de $\mathrm{m}^{3} \mathrm{año}^{-1}$.

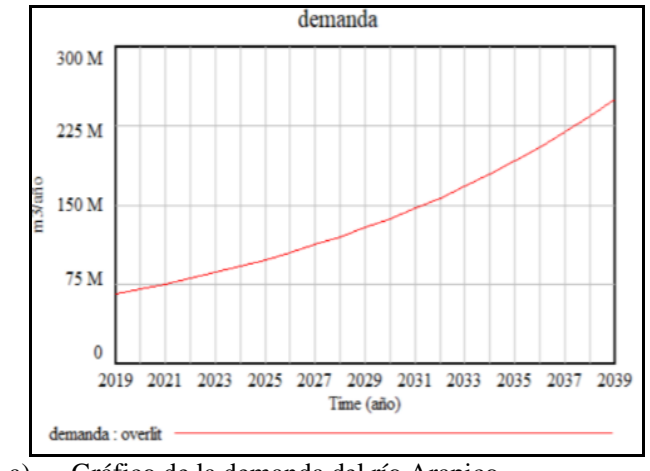

a) Gráfico de la demanda del río Arapico

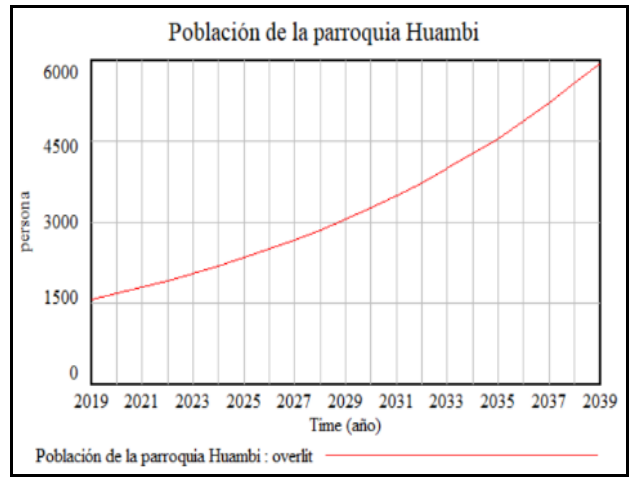

b) Población de la parroquia Huambi

Fig. 5 Relación entre la población dedicada a la agricultura y la demanda. (Fuente: los autores)

Así como el determinar las variables condicionantes para la presencia del líquido vital como precipitación, la evapotranspiración y los aportes de las principales redes hídricas afluentes al sistema. Así como también, se calculó un aporte de agua neta de 7072726464 metros cúbicos por año como punto de partida en el año 2019, con una población inicial de habitantes y con un consumo per cápita de 42032.5 metros cúbicos por habitante por año. En definitiva, se determinó que la cantidad de agua (oferta) no satisface las necesidades de la población durante los 20 años establecidos para la modelación, establecido como tiempo final para el año 2039, para lo cual en los años donde no satisfaga la necesidad de agua para cultivo se recomienda realizar planes de manejo y conservación de las principales fuentes hídricas que conforman el Río Arapico con el propósito de satisfacer las necesidades futuras, también el control de fugas en las redes de distribución en el sector agrícola y del agua a los hogares, sin embargo no deja de ser importante desarrollar campañas de concienciación y uso racional del mismo. 

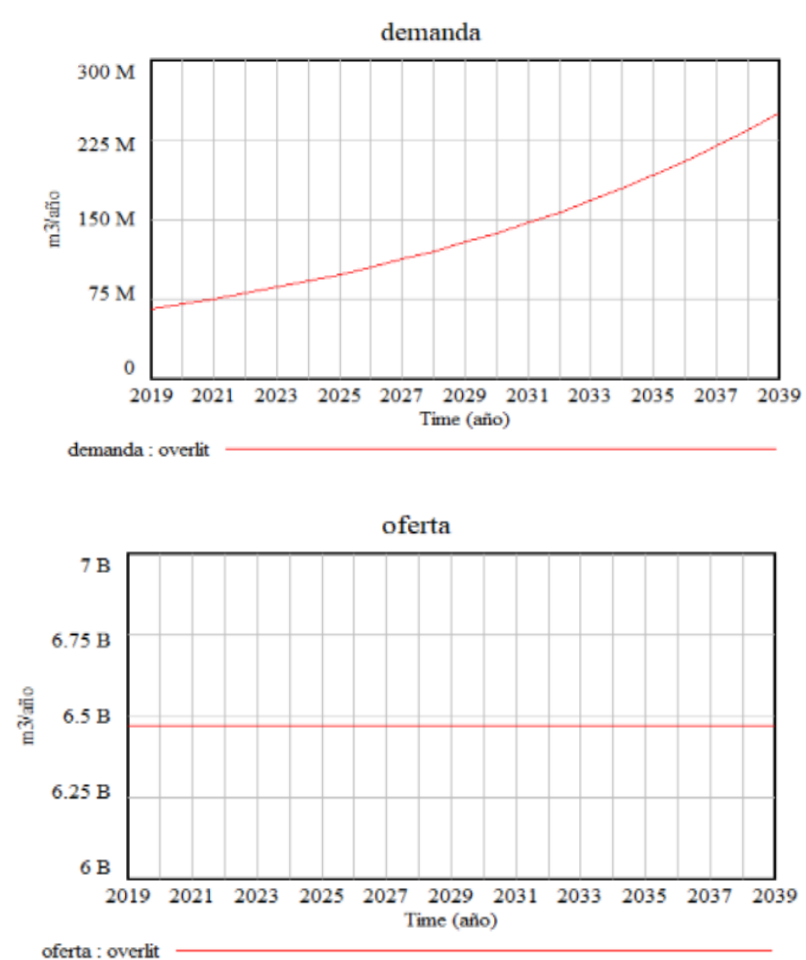

Fig. 6 Relación entre la oferta y la demanda. (Fuente: Los autores

\section{CONCLUSIONES}

Se determinó que el área de estudio tiene una baja probabilidad de intercambio de sodio, el índice de salinidad potencial es bajo, tiene baja conductividad y concentración de sales disueltas, lo que lo convierte en un río apto para la actividad agrícola.

Así mismo, se determinó que el río tiene una mayor correlación con los compuestos de bicarbonato de calcio que con los de magnesio, y la mejor correlación se obtuvo al comparar el RAS con la conductividad eléctrica con una correlación de 0.8596 , esos fueron los datos más interesantes dentro las correlaciones realizadas. Ante un déficit en los recursos hídricos del río Arapicos pertenecientes a la microcuenca Tutanangoza, a través de un modelo de simulación de oferta y demanda, se observó que a partir de 2021, se implementarían los respectivos planes de contingencia de manejo y conservación.

Las principales fuentes de agua que conforman el río Arapicos para satisfacer las necesidades futuras, deteniendo la posible escasez, porque los resultados de las simulaciones también muestran que, en diferentes escenarios, esta situación puede anticiparse mediante la toma de medidas que pueden ocurrir.

\section{REFERENCIAS}

[1] J. A. Tercedor and A. S. Ortega, "Un método rápido y simple para evaluar la calidad biológica de las aguas corrientes basado en el de hellawell (1978)," Limnética, vol. 4, no. 1978, pp. 51-56, 1978.

[2] J. Alba-Tercedor, "Macroinvertebrados Acuáticos y Calidad de las Aguas de los Ríos.," IV Simp. del Agua en Andalucía, vol. II, no. september, p. Almeria, 203-213, 1996.

[3] J. (Jean) Rodier and C. Geoffray, Análisis de las aguas: aguas naturales, aguas residuales, agua de mar; química, fisicoquímica, bacteriología, biología, 2,ilustrad ed. Omega, 1981.

[4] D. Carrera, D. Guevara, P. Tamayo, L. Guallichico, "Análisis multivariado de las aguas de la Subcuenca del Río Ambi en época de estiaje y su relación con la calidad desde el punto de vista agrícola," Congr. Cienc. y Tecnol. ESPE, vol. 3, no. september, pp. 123-129, 1981.

[5] W. J. F. Millán , J. Mathison, M. Alvares and Instituto, "Estudio comparativo de la dureza del agua en el estado Mérida y algunas localidades del centro y occidente de Venezuela," Cienc. e Ing., vol. 24, no. Mérida, pp. 39-46, 2003.

[6] J. G. T. Díaz, "Importancia de los ríos en el entorno ambiental," Rev. del Inst. Investig. la Fac. Geol. Minas, Metal. y Ciencias Geográfica, vol. 3 , no. $5,200 \mathrm{AD}$.

[7] Global Water Partnership (GWP), "Situación de los recursos hídricos en Centroamérica: hacia una gestión integrada," Glob. Water Partnersh. Cent. Am., p. 100, 2017.

[8] S. Miyamoto, L. Fenn, and D. Swietlik, "Flow, salts, and trace elements in the Rio Grande: a review," in Texas Water Resource, C. Station, Ed. Texas Agriculture Experimental Station-Texas Water Resources Institute, pp. 1-18, 1995.

[9] D. V. Carrera Villacrés, "Salinidad en suelos y aguas superficiales y subterráneas de la cuenca evaporítica de Río Verde-Matehuala, San Luis Potosí.," Colegio de Postgraduados (COLPOS), 2011.

[10] M. Irfan, M. Arshad, A. Shakoor, and L. Anjum, "Impact of irrigation management practices and water quality on maize production and water use efficiency," J. Anim. Plant Sci., vol. 24, no. 5, pp. 1518-1524, 2014.

[11] FAO, "Uso de agua en riego," AQUASTAT, 2016. [Online]. Available: http://www.fao.org/nr/water/aquastat/water_use_agr/indexesp.stm [Accessed: 31-May-2019].

[12] E. P. Mayorga Llerena, "Purificación de aguas de regadio del canal Latacunga-Salcedo-Ambato por filtración anaeróbica-aeróbica," Universidad de las Fuerzas Armadas ESPE, 2014.

[13] H. GAD, "Plan de Desarrollo y Ordenamiento Territorial de la Parroquia Huambi," pp. 0-108, 2012.

[14] M. S. GAD, “Actualización del diagnóstico del PDOT," 2011.

[15] Gad Parroquial Logroño, "Plan De Desarrollo y Ordenamiento Territorial del cantón Logroño," pp. 1-147, 2012.

[16] J. P. Flores Cárdenas, "Deserción escolar en adolescentes embarazadas de la parroquia Huambi del cantón Sucúa, en el año lectivo 2014 2015," Universidad del Azuay, 2016.

[17] S. Dwivedi, "Symposium on Colossal Data Analysis and Networking (CDAN)," Compr. Study Data Anal. Tools, no. March, pp. 1-8, 2016.

[18] J. Frejio Bouso, "El paquete estadístico R by matilez - issuu," Cuadernos Metodológicos, 48, 2013. [Online]. Available: https://issuu.com/matilez/docs/el_20paquete_20estadistico_20r. [Accessed: 31-May-2019].

[19] L. S. C. and A. D. E. Arnold E. Greenberg, "APHA Method 4500-O."

[20] L. S. C. and A. D. E. Arnold E. Greenberg, "APHA Method 2550," Stand. Methods Exam. Water Wastewater, vol. 552, 1992.

[21] N. M. Nmx-aa--, A. D. E. Agua, and D. D. E. Arsenico, "Norma mexicana nmx-aa-46-1981, analisis de agua determinacion de arsenico," pp. 1-5, 2009.

[22] Eaton A Clesceri L Greenberg A Franson M, "APHA Method 2510," Stand. Methods Exam. Water Wastewater, vol. 552, 1992.

[23] L. S. C. and A. D. E. Arnold E. Greenberg, "APHA Method 3500-Ca," vol. 552, 1992.

[24] L. S. C. and A. D. E. Arnold E. Greenberg, "APHA Method 3500-Mg," Methods Exam. Water Wastewater., vol. 552, 1992.

[25] L. S. C. and A. D. E. Arnold E. Greenberg, "APHA Method 4500-SO4- 
2," Stand. Methods Exam. Water Wastewater, no. 4, 1992.

[26] L. S. C. and A. D. E. Arnold E. Greenberg, "APHA Method 2330," Water Environ. Fed., vol. 552, no. Washinfton,DC, 1992.

[27] T. Tartabull Puñales and R. C. Aguilar Betancourt, "La calidad del agua para el riego. Principales indica- dores de medida y procesos que la impactan," Rev. Científica Agroecosistemas, vol. 4, no. 1, pp. 47-61, 2016.

[28] G. Dercon, "Zonificacion Agroecologica del Austro Ecuatoriano," Cuenca, p. 148, Aug-1998.

[29] FAO, "Guía para la descripción de suelos," Organ. las Nac. Unidas para la Aliment. y la Agric., vol. 3, no. 4, p. 100, 2009.

[30] C. Gasca, J. Menjivar, and A. Torrente, "Cambio en el porcentaje de sodio intercambiable y RAS de un suelo," Acta Agron., vol. 60, no. 1, pp. 27-38, 2011.

[31] D. Carrera, "Calidad de las aguas para riego y metamorfosis de las aguas naturales," 2018. [Online]. Available: https://www.researchgate.net/publication/326831496_Calidad_de_las_a guas_para_riego_y_metamorfosis_de_las_aguas_naturales [Accessed: 31-May-2019].

[32] D. Carrera Villacrés, E. Guerrón Varela, L. Cajas Morales, T. González Farinango, É. Guamán Pineda, and P. A. Velarde Salazar, "Relación de temperatura, $\mathrm{pH}$ y $\mathrm{CE}$ en la variación de concentración de fosfatos en el Río Grande, Cantón Chone," Congr. Cienc. y Tecnol. ESPE, vol. 13, no. 1,2018 . 